\title{
Access and Visualize Oceanographic Data from International Project's Databases and Marine Hydrophysical Institute Oceanographic Data Bank Using the Ocean Data View Software
}

\author{
Elena V. Zhuk \\ Marine Hydrophysical Institute, Russian Academy of Sciences, Sevastopol, Russia \\ alenixx@gmail.com
}

\begin{abstract}
The paper provides a brief overview of the processes for collecting, archiving and accessing oceanographic insitu data from international projects such as SeaDataNet[1], EMODNET[2], and BlackSeaScene[3]. Using SeaDataNet as an example, the structure of a distributed data network is described and the procedure for selecting and downloading oceanographic data from the SeaDataNet portal is considered. Particular, attention is paid to the free software Ocean Data View (ODV) [4] providing a user-friendly interface for working with the downloaded oceanographic data. Moreover, the paper considers the Regional Geographic Information System (GIS) for the Black Sea developed at the Marine Hydrophysical Institute (MHI) of the Russian Academy of Sciences, providing online access and visualization of oceanographic data of the MHI Oceanographic Data Bank (BOD MHI) [5,6].
\end{abstract}

Keywords: SeaDataNet, SeaDataCloud, ODV, oceanographic data, GIS «Black Sea», BOD MHI, online data access. 


\title{
ДОСТУП И ВИЗУАЛИЗАЦИЯ ДАННЫХ МЕЖДУНАРОДНЫХ ПРОЕКТОВ И ДАННЫХ БАНКА ОКЕАНОГРАФИЧЕСКИХ ДАННЫХ МГИ В ПРОГРАММЕ ODV
}

\author{
E.B. Жyк \\ Морской гидрофизический институт РАН, Севастополь, Россия \\ alenixx@gmail.com
}

\begin{abstract}
Данная работа посвящена краткому обзору механизмов сбора, хранения и доступа к океанографическим данным контактных измерений в международных проектах, таких как SeaDataNet [1], EMODNET [2], BlackSeaScene [3]. На примере SeaDataNet описана структура распределенной сети проекта и рассмотрена процедура выбора и загрузки океанографических данных с портала. Особое внимание уделено свободно распространяемому программному продукту Ocean Data View (ODV) [4], предоставляющему дружественный интерфейс и мощный инструментарий для работы с океанографическими данными. В работе так же рассматривается разрабатываемая в Морском гидрофизическом институте (МГИ) РАН региональная геоинформационная система (GIS) по Чёрному морю, обеспечивающая онлайн доступ и визуализацию океанографических данных Банка океанографических данных (БОД) МГИ $[5,6]$.
\end{abstract}

Ключевые слова: SeaDataNet, SeaDataCloud, ODV, океанографические данные, ГИС «Чёрное море», БОД МГИ, онлайн доступ к данным.

\section{Введение}

В настоящее время быстрого развития вычислительной техники, информационных технологий и средств коммуникации большое значение приобретают распределенные информационные системы для хранения и доступа к данным. Существует ряд международных (европейских) проектов, в рамках которых ведутся работы по сбору, хранению и обеспечению online доступа к данным. К таким проекта относятся Black SeaScene и UP-GRADE BlackSeaScene, серия проектов EMODNET и проекты SeaDataNet, с 2016 г. -- SeaDataCloud.

Подробная информация об этих проектах, участниках и особенностях каждого проекта доступна на сайтах проектов и в многочисленных руководствах. Здесь лишь остановимся на их общей части - организации сбора, хранения и процедуре доступа к данным на приме SeaDataNet.

Информационные системы и базы океанографических данных создаются не только в рамках международных проектов, но и в исследовательских институтах. Примером такой информационной системы является ГИС "Чёрное море", разрабатываемая в МГИ РАН и обеспечивающая доступ к Банку океанографических данных (БОД) МГИ [5,6]. 


\section{Структура SeaDataNet}

Проект SeaDataNet представляет собой распределенную инфраструктуру для хранения, управления и обеспечения доступа к большому количеству разнообразных океанографических данных контактных измерений. Сами данные хранятся на серверах Центров данных, участвующих в проекте, а соответствующее им описание данных (метаданные) находится на портале SeaDataNet.

Центры данных с помощью специального программного обеспечения MIKADO [7] для каждой океанографической станции генерируют метаданные (Common Data Index ) в формате XML и отправляют их техническому координатору проекта (MARIS). MARIS проверяет метаданные и размещает их на портале. Для унификации описания параметров измерений, единиц измерений, приборов и др. Британским океанографическим центром данных (BODC) [8] была разработана система словарей. Эти словари автоматически подгружаются в MIKADO и используются при генерации метаданных и соответствующих им данных. Каждому Common Data Index (CDI) ставится в соответствие набор океанографических данных, который размещается на сервере Центра данных в специальном программном обеспечении Download Manager (DM), которое обеспечивает корректное обращение к данным на локальном сервере. Соответствие между метаданными и данными описывается в структурированном текстовом файле coupling table, хранящемся на локальном сервере. Данные на локальном сервере Центра данных могут быть представлены как в виде текстовых файлов в формате ODV, так и в виде баз данных, к которым описаны процедуры доступа и запросы.

На рисунке 1 представлены основные компоненты системы и их взаимодействия.

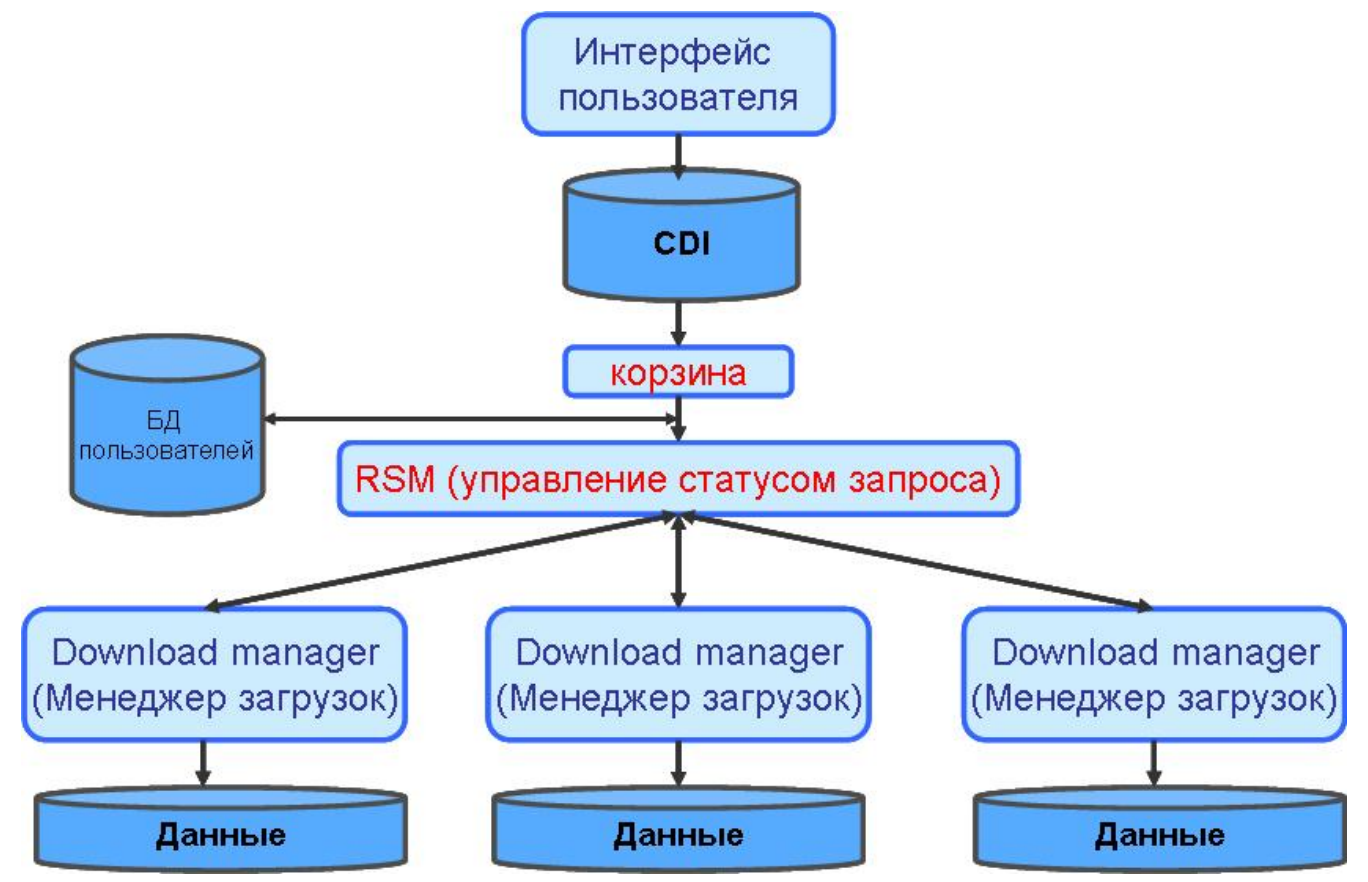

Puс. 1. Структура распределенной системы SeaDataNet

Запросы к данным формируются в Интерфейсе пользователя, и выбранные метаданные (CDI) помещаются в корзину. Затем происходит авторизация пользователя, и запрос передается в модуль управления статусами запросов, где запрос обрабатывается и отправляется в соответствующий центр данных, в котором хранятся запрашиваемые данные.

На рисунке 3 представлена схема взаимодействия между пользователем, порталом SeaDataNet и центрами данных. 
DM, установленный на локальном сервере, взаимодействует с диспетчером состояния запросов и отслеживает состояние готовности и отправки запрошенных данных пользователю.

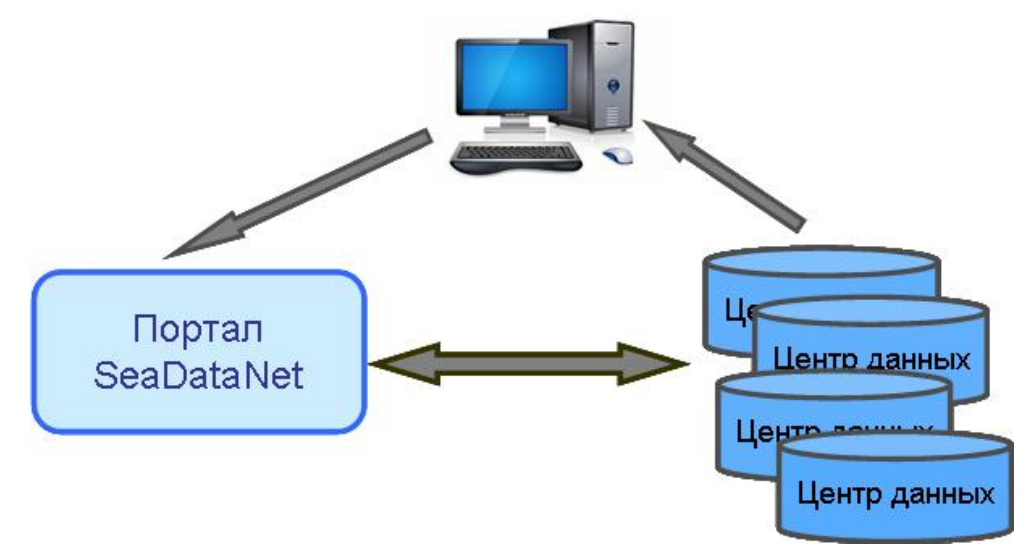

Рис. 3. Схема взаимодействия между пользователем, порталом SeaDataNet и центрами данных

\section{Поиск данных и их загрузка на портале SeaDataNet}

Портал SeaDataNet предоставляет удобный интерфейс для поиска и скачивания данных. Этот процесс состоит из следующих шагов, представленных на рисунке 4.

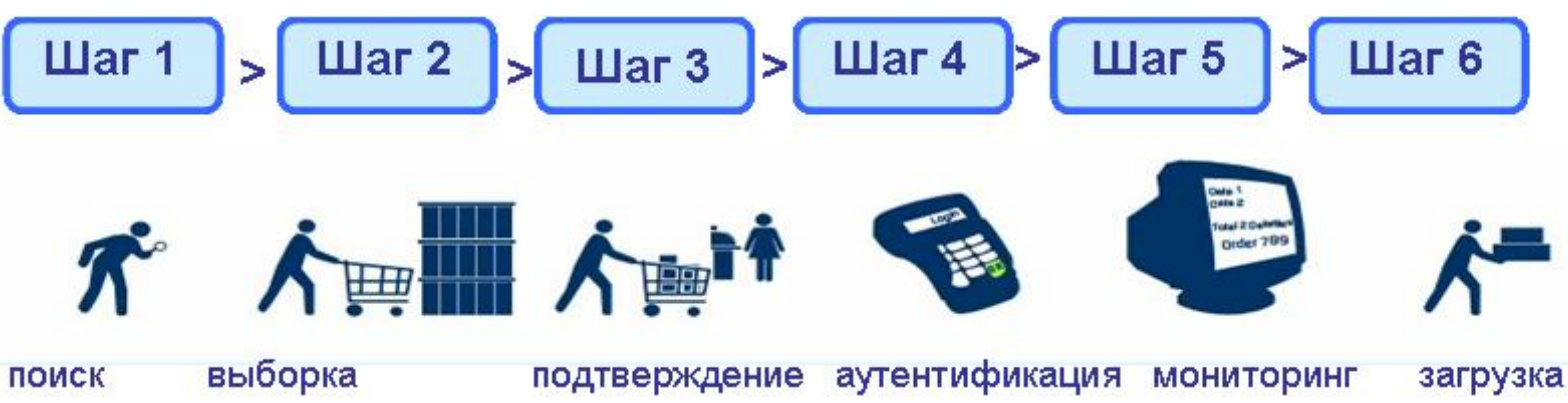

Pис.4. Этапы поиска и загрузки океанографических данных с портала SeaDataNet.

1. Поиск данных по метаданным. Интерфейс пользователя позволяет задать ряд фильтров, таких как географический охват интересующей области - координаты или название, владелец данных, участник проекта, представляющий данные, тип данных, прибор измерений, временной охват и пр.

2. Отправка выбранных данных в корзину.

3. Подтверждение выборки.

4. Авторизация пользователя и обработка запроса системой RSM (управление статусом запроса).

5. Мониторинг обработки запроса. На этом этапе происходит обращение к соответствующим локальным серверам и формирование файлов с запрошенными данными в выбранном формате (ODV или NetCDF)

6. Передача пользователю ссылки для скачивания на запрошенные данные. 
Этот же механизм организации распределенного хранения данных и обеспечения доступа к ним используется в проектах BlackSeaScene, Up-grade BlackSeaScene и EMODNET.

На рисунке 5 представлены изображения пользовательского интерфейса для каждого этапа поиска и загрузки данных.

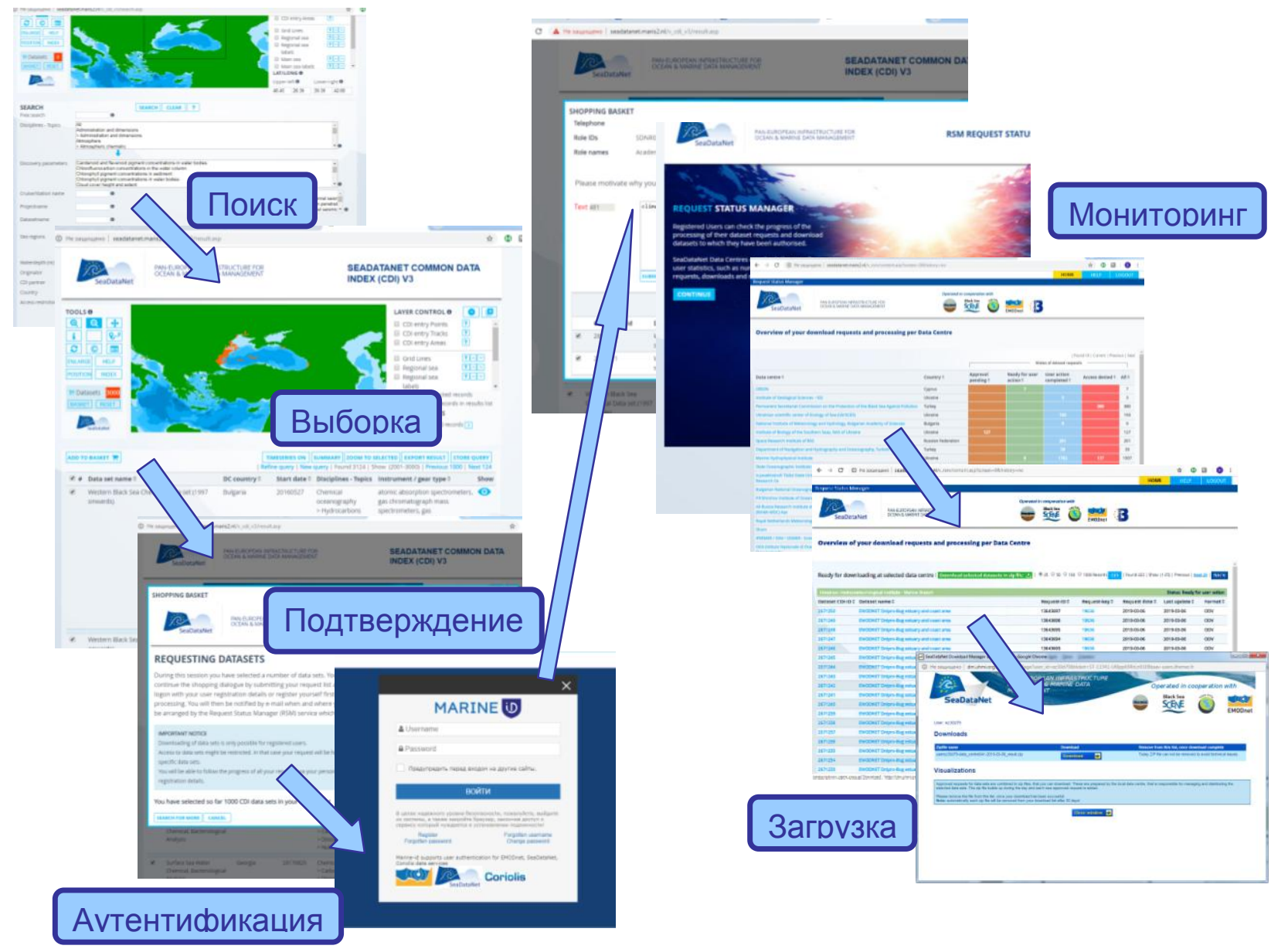

Рис. 5. Пользовательский интерфейс на каждом этапе поиска и загрузки данных.

\section{Ocean Data View}

Портал SeaDataNet позволяет скачивать данные в формате Ocean Data View. Этот формат представляет собой структурированный текстовый файл предназначенный для хранения рейсовых данных. В верхней части, так называемой шапке, может содержаться любая дополнительная информация о рейсе, измерениях, проекте, руководителе и пр. Затем идет обязательная строка //sdn_parameter_mapping и строки, описывающие соответствия между параметрами, находящимися в файле, и словарями. Параметры содержат обозначения в кодах словарей и соответствующее им название столбца. Затем идет пустая строка // и заголовок, который обязательно содержит столбцы: Рейс, Станция, Тип, Дата:Время, Долгота, Широта, Идентификатор_CDI, Код организации, Глубина, Главная переменная (в профилях станций глубина измерений) и столбцы с измеренными параметрами. Подробное описание формата приведено в руководстве пользователя программы ODV [4]. На рисунке 6 приведен вид файла в формате ODV. 


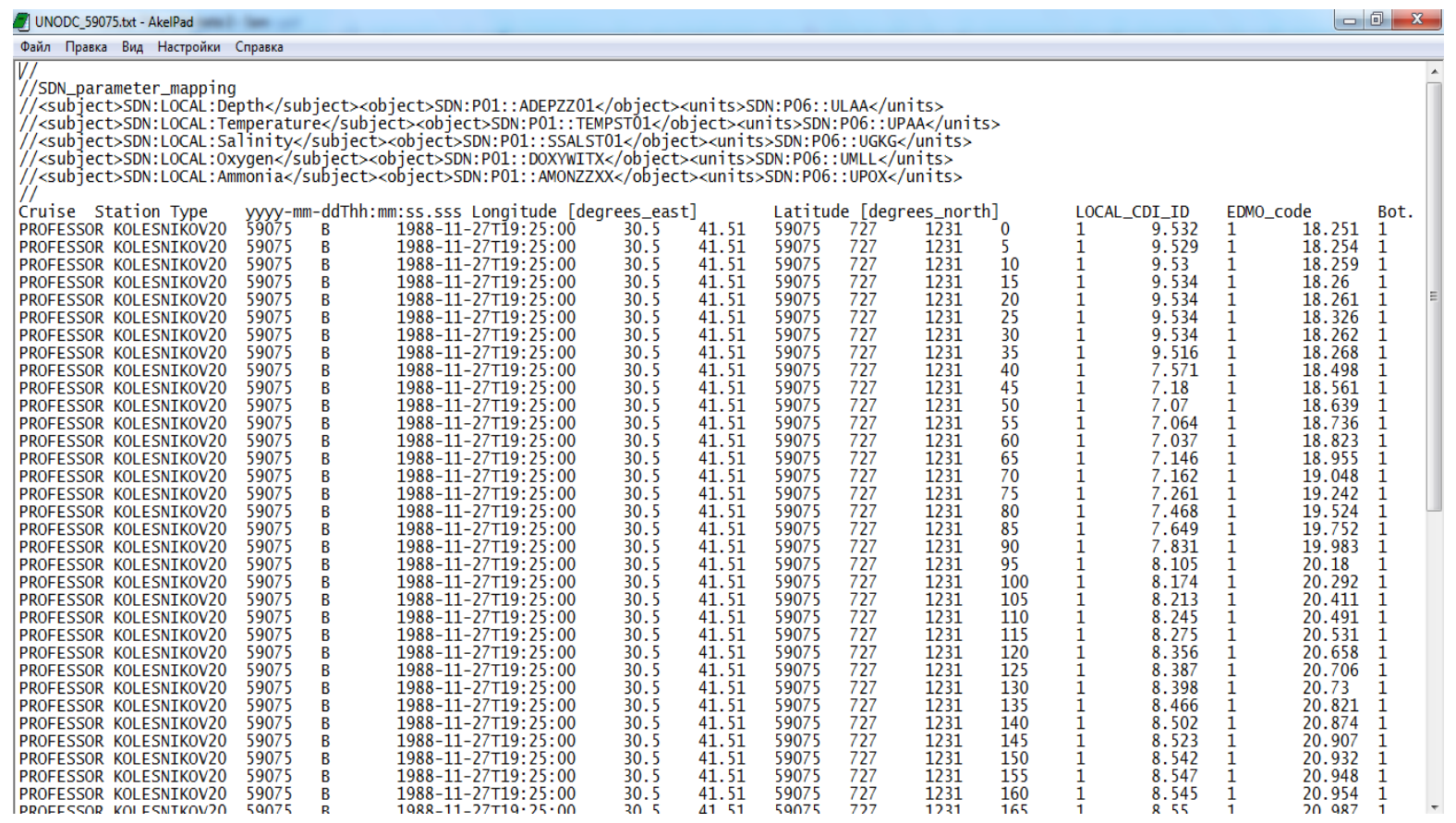

Puс. 6. Файл в формате $O D V$

Для работы с файлами в формате ODV можно использовать различные программы - от блокнота до собственных разработок, но одной из наиболее удобных программ является ODV, которая была разработана специально для этого формата в рамках проекта SeaDataNet доктором Рейнером Шлицером из института AWI [4]. Эта программа является свободно распространяемой, имеет удобный интерфейс и обладает мощным функционалом. С её помощь можно отображать положение данных на карте, строить профили, поверхности, разрезы, делать интерполяцию, строить изолинии, проводить контроль качества данных и многое другое. На рисунке 7 приведен пример визуализации данных станций 29-го рейса НИС «Профессор Колесников».

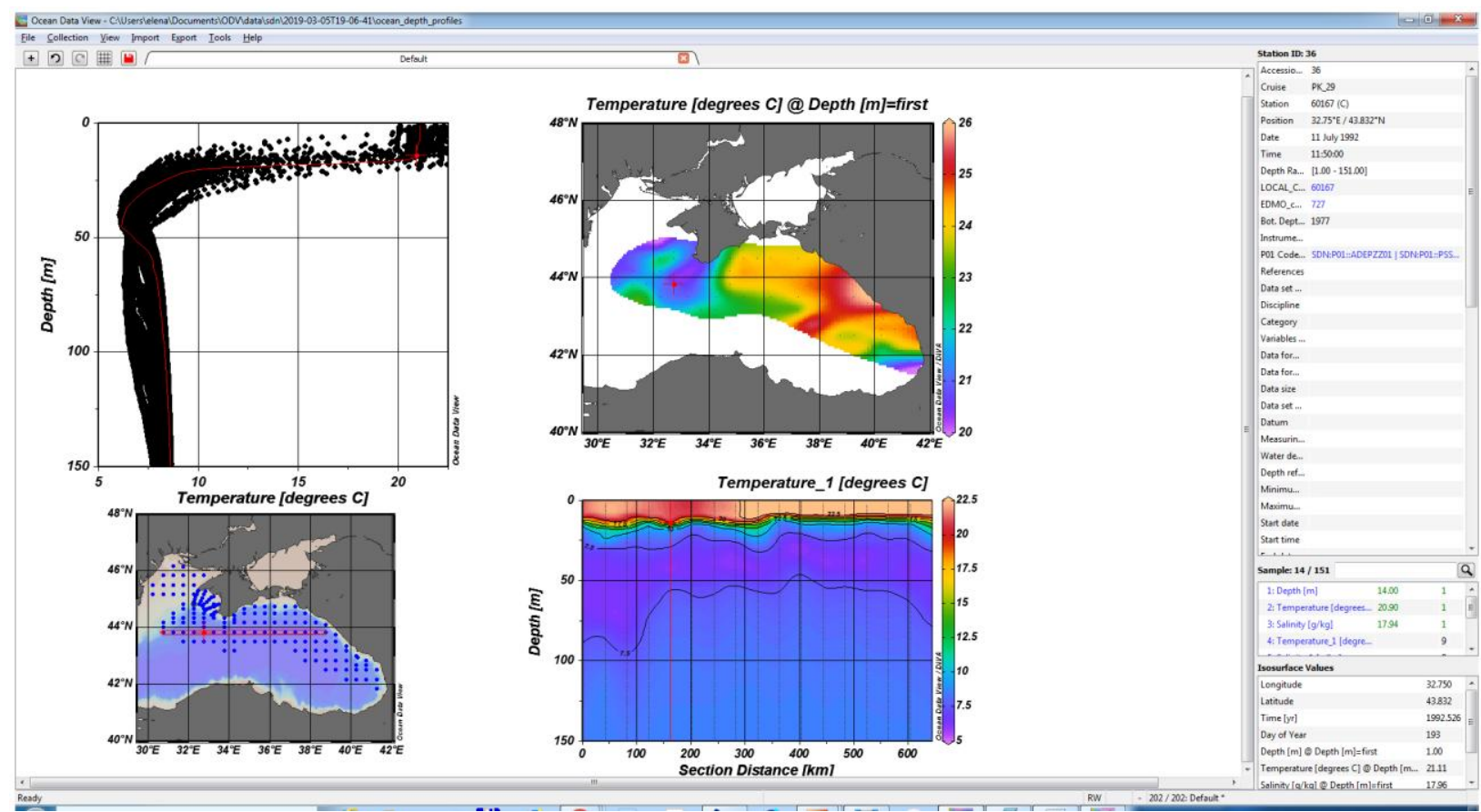

Рис. 7. Визуализащия данных в $O D V$ 


\section{ГИС «Чёрное море»}

В МГИ РАН ведутся работы по обеспечению онлайн доступа и визуализации океанографических данных, хранящихся в БОД МГИ [5,6].

ГИС «Чёрное море» создана на базе клиент-серверной архитектуры. Функционал пользовательского интерфейса реализован с использованием библиотек jQuery и OpenLayers. Серверная часть представляет собой Арасhe web server с интегрированным Mapserver, который используется в качестве картографического сервиса. Океанографические данные хранятся в реляционной БД, реализованной на MySQL. Запросы от пользовательского интерфейса к БД обрабатываются рhр скриптами, полученные результаты визуализируются в качестве карт, таблиц и профилей. Данная система обеспечивает выборку океанографических данных, построение профилей, отображение карт климатического атласа и спутниковых изображений MODIS AQUA по Чёрному морю. Выбранные данные можно скачать в формате ODV и в дальнейшем работать с ними на локальном компьютере в том числе и с помощью программы ODV. На рисунке 8 представлен пользовательский интерфейс ГИС «Чёрное море» с выбранными данными.

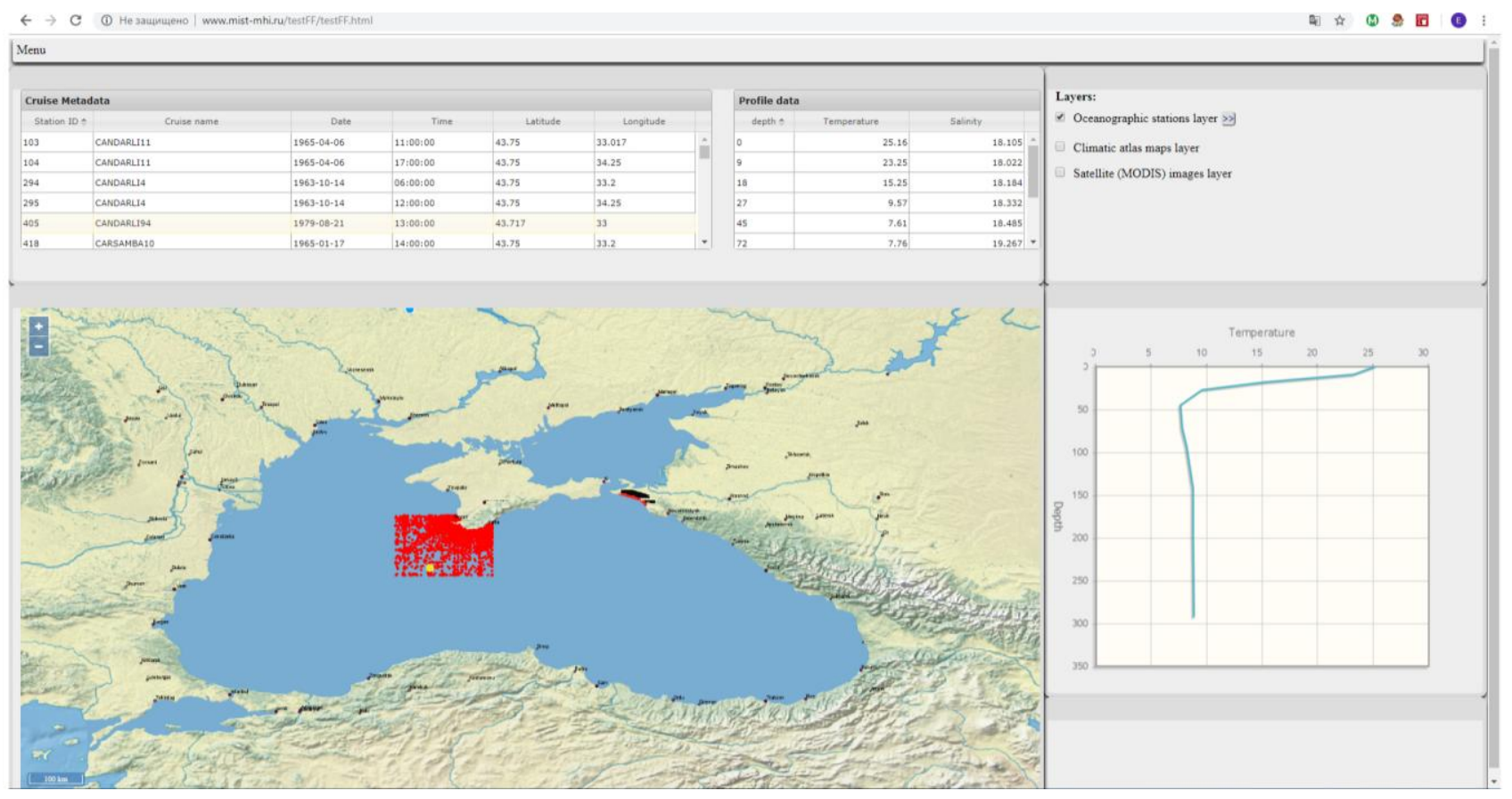

Рис. 8. Пользовательский интерфейс ГИС «Чёрное море»

\section{Заключение}

В современном научном сообществе существуют проекты, в рамках которых создаются океанографические информационные системы, предоставляющие доступ к большому количеству разнообразных океанографических данных, хранящихся более чем в 50 центрах данных через единый интерфейс. Эти данные могут быть использованы в научных исследованиях не только участниками проекта, но и практически любыми учеными, работающими в области океанологии и в смежных областях. Удобным инструментом обработки и визуализации этих данных является бесплатное программное обеспечение ODV. 


\section{Благодарности}

Данная работа ведется в рамках тем госзаданий ФГБУН МГИ 0827-2018-0002 (Оперативная океанология) и 0827-2018-0004 (Прибрежные исследования).

\section{References}

[1] SeaDataNet https://www.seadatanet.org/

[2] EMODNET http://www.emodnet.eu/

[3] BlackSeaScene http://www.blackseascene.net/

[4] Ocean Data View http://odv.awi.de/

[5] Zhuk E., Khaliulin A., Zodiatis G., Nikolaidis A., Isaeva E., Black Sea GIS developed in MHI, Proc.

SPIE 9688, Fourth International Conference on Remote Sensing and Geoinformation of the Environment (RSCy2016), 96881C (August 12, 2016); doi: 10.1117/12.2241631 Volume 9688

http://proceedings.spiedigitallibrary.org/proceeding. aspx? articleid $=2545474$

[6] Zhuk E., Khaliulin A., Ingerov A, Geoinformation system "Black Sea”, Ecologicheskaya bezopasnost v pribrejnoy I shelfovoy zon moray, 2016, 4, pp. 83-87. (In Russian).

[7] MIKADO https://www.seadatanet.org/Software/MIKADO

[8] BODC https://www.bodc.ac.uk/ 